\title{
Novel Synthesis of Fused Pyrazolopyrimidines and C-Nucleosides of Thienopyrimidone with Expected Antimicrobial Activity
}

\section{H.A.R. Hussein*, Kh. M. Abu-Zied and A.A. Abu-Hashem}

Department of Photochemistry (Heterocyclic Unit), National Research Centre, Dokki, Cairo, Egypt.

\begin{abstract}
S TARTING from thienopyrimidine hydrazine derivatives $(1 \mathrm{a}, \mathrm{d})$ $\mathcal{N}$ and $(2 \mathrm{~b}, \mathrm{c})$ a series of pyrazolopyrimidine derivatives has been synthesized. Also, some acyclic and cyclic C- nucleosides of thienopyrimidone (7-10) were prepared by treating compound 1a with aldoses. The prepared products showed antimicrobial activity. Structures of the new compounds were elucidated on the basis of their elemental analyses and spectral data.
\end{abstract}

Keywords: Pyrazolopyrimidine, C-Nucleosides, NMR spectra, Mass spectra, Antibacterial and Antifungal Activities.

In the recent years the pyrazolo-, and fused pyrazolopyrimidine compounds have attracted great attention due to their diverse properties ${ }^{(1-5)}$, biological ${ }^{(6,7)}$, herbicide and bactericide activities ${ }^{(8,9)}$. These facts prompted us to prepare new fused pyrimidine derivatives at 1-position of the pyrazole ring to obtain new compounds with expected antimicrobial activity ${ }^{(10-13)}$.

\section{Results and Discussion}

Compound 1a,d or 2b,c was refluxed with [bis (methylthio) methylene] malononitrile in methanol to afford the corresponding substituted pyrazole derivatives (3a-d) (Scheme 1). Structures of the latter compounds were confirmed with their elemental analyses and spectral data (Exp). The IR spectra of $3 \mathrm{a}-\mathrm{d}(\mathrm{KBr}) \mathrm{cm}^{-1}$ showed $\left(\mathrm{NH}_{2}\right)$ around 3306 and $(\mathrm{CN})$ around 2224 ; ${ }^{1} \mathrm{H}-\mathrm{NMR}$ of $3 \mathrm{a}\left(\mathrm{DMSO}-d_{6}\right)$ as an example, showed signals $\delta$ ppm at: $2.54\left(\mathrm{~s}, 3 \mathrm{H}, \mathrm{CH}_{3}\right)$, $2.66\left(\mathrm{~s}, 3 \mathrm{H}, \mathrm{SCH}_{3}\right), 7.51-7.60(\mathrm{~m}, 5 \mathrm{H}, \mathrm{Ar}-\mathrm{H}), 6.31\left(\mathrm{~s}, 2 \mathrm{H}, \mathrm{NH}_{2}, \mathrm{D}_{2} \mathrm{O}\right.$ exchangeable) and $9.02\left(\mathrm{~s}, 1 \mathrm{H}, \mathrm{NH}, \mathrm{D}_{2} \mathrm{O}\right.$ exchangeable)

The synthesis of pyrazolo[3,4- $d]$ pyrimidin-4-one derivatives (4a-d) was achieved by refluxing compounds $3 \mathrm{a}-\mathrm{d}$ with formic acid in presence of catalytic amounts of hydrochloric acid. The IR spectra of the latter compounds showed absence of $\mathrm{CN}$ and $\mathrm{NH}_{2}$ groups and the presence of $\mathrm{OH}$ group absorption. Their mass spectra gave the molecular ion peaks as the base peak in each case. (See experimental).

*E-mail: hodarf@hotmail.com 


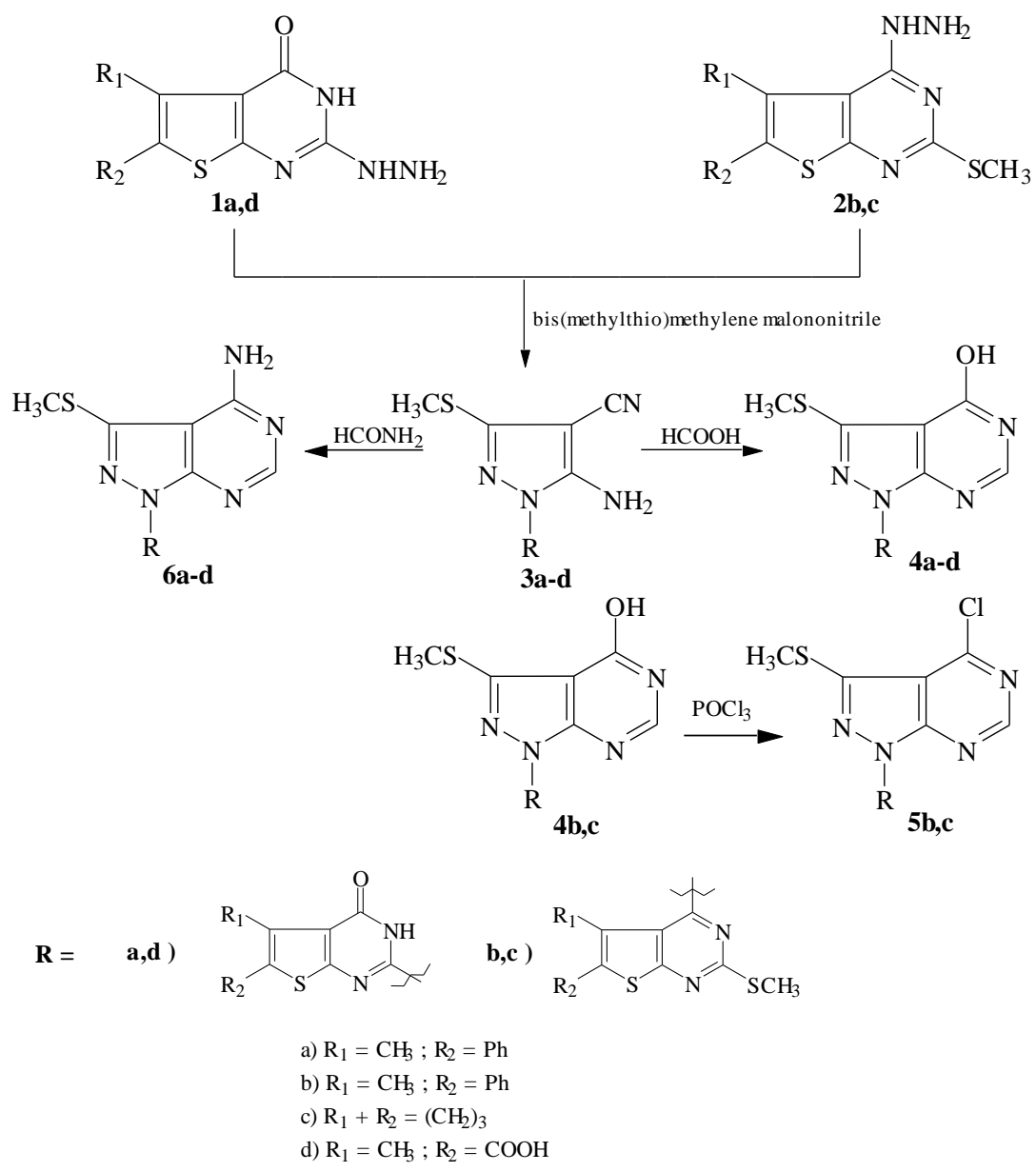

Scheme 1

Compounds $(4 \mathrm{~b}, \mathrm{c})$ were converted into their corresponding 4-chloro derivatives $(5 \mathrm{~b}, \mathrm{c})$ by refluxing with phosphorus oxychloride. The IR spectra showed absence of $\mathrm{OH}$ group. Their MS gave characteristic fragmentation pattern confirming the presence of one $\mathrm{Cl}$ atom in their molecules (Scheme 1).

When compounds 3a-d were refluxed with formamide they gave 4aminopyrazolopyrimidine derivatives (6a-d). The IR spectra of the latter compounds showed absence of $\mathrm{CN}$ group and the presence of $\mathrm{NH}_{2}$ groups; ${ }^{1} \mathrm{H}-\mathrm{NMR}$ of $6 \mathrm{~b}$ $\left(\mathrm{DMSO}-d_{6}\right)$, as an example, showed signals $\delta$ ppm at: $2.53\left(\mathrm{~s}, 3 \mathrm{H}, \mathrm{CH}_{3}\right), 2.57(\mathrm{~s}$, $\left.3 \mathrm{H}, \mathrm{SCH}_{3}\right), 2.60\left(\mathrm{~s}, 3 \mathrm{H}, \mathrm{SCH}_{3}\right), 5.2\left(\mathrm{~s}, 2 \mathrm{H}, \mathrm{NH}_{2}, \mathrm{D}_{2} \mathrm{O}\right.$-exchangeable), 7.50-7.54 (m, $5 \mathrm{H}, \mathrm{Ar}-\mathrm{H})$, and $8.00(\mathrm{~s}, 1 \mathrm{H}, \mathrm{CH})$.

Egypt. J. Chem. 53, No. 4 (2010) 
The C-nucleosides (7a-c), (Scheme 2), were prepared by reacting compound 1a with some aldoses, namely, D-glucose, D-mannose and D-arabinose in boiling dioxane in the presence of catalytic amounts of piperidine. The isolated products showed the absence of amino group in the IR spectra and their ${ }^{1} \mathrm{H}-\mathrm{NMR}$ spectra (DMSO- $d_{6}$ ) exhibited, in each case, characteristic signal due to $-\mathrm{CH}=\mathrm{N}-$ methane proton of shiff's base . In addition, the spectra showed signals due to protons of the sugar residue. (See Experimental).

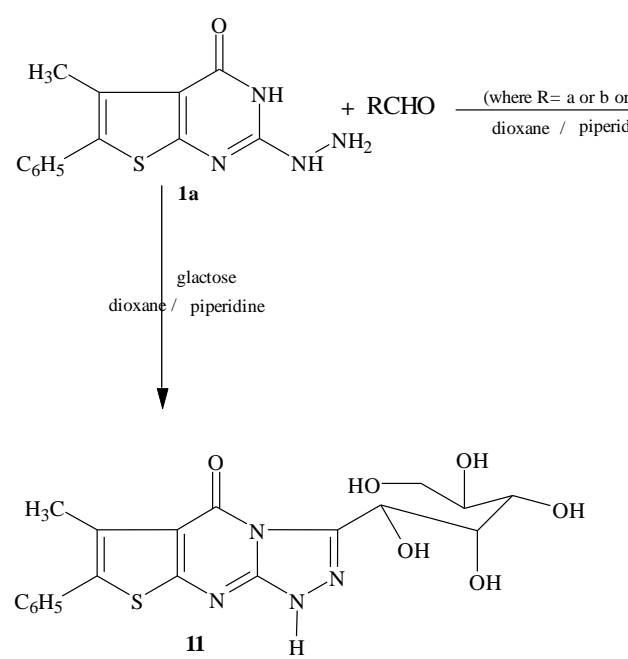

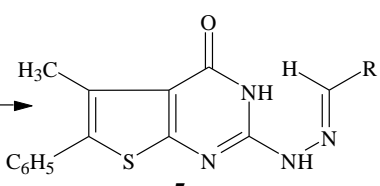<smiles>CC(C)CC(C)C</smiles>

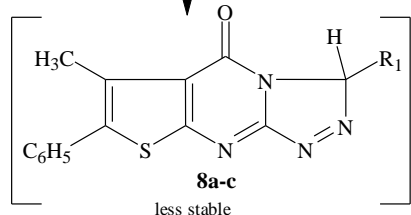

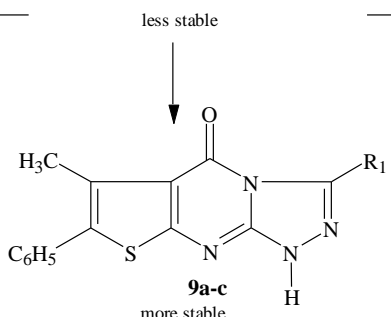
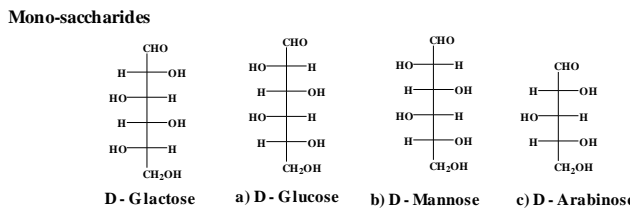

where $\mathbf{R}=$
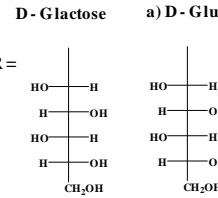

b) D- Mannose c) D - Arabinos<smiles>[R]c1n[nH]c2nc3sc(CCCCC)c(C)c3c(=O)n12</smiles>

(a)

(b)

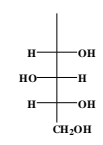

$10 \mathrm{a}-\mathrm{c}$

(c)

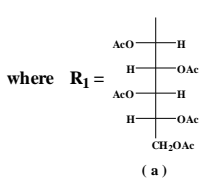
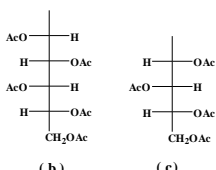

Scheme 2

Cyclization of the hydrazone derivatives (7a-c) are achieved by acetylation to afford acetylated derivatives $(9 a-c)$. (See Experimental). The IR spectra of the

Egypt. J. Chem. 53, No. 4 (2010) 
latter compounds showed absence of $\mathrm{OH}$ groups and the presence of the $\mathrm{CO}$ acetyl groups. The ${ }^{1} \mathrm{H}-\mathrm{NMR}$ spectra in $\mathrm{CDCl}_{3}$ showed the presence of acetyl groups, one exchangeable $-\mathrm{N} \underline{H} N=\mathrm{C}^{-}$proton and absence of $-\mathrm{C} \underline{H}=\mathrm{N}-$ methine proton.

Finally, the deprotection of the acyclic $C$-nucleosides (9a-c) are achieved by stirring in methanolic sodium methoxide at room temperature to give the acyclic $C$-nucleosides (10a-c). Structures of the latter compounds were confirmed on the basis of their spectral data. The products revealed absorption bands for $(\mathrm{OH}$ and $\mathrm{NH}$ ) in their IR spectra while their ${ }^{1} \mathrm{H}-\mathrm{NMR}$ spectra showed singals of hydroxyl groups (exchangeable with $\mathrm{D}_{2} \mathrm{O}$ ). (See Experimental).

On the other hand, compound 11 was prepared directly by reacting compound 1a with D-galactose in boiling dioxane in the presence of a catalytic amount of piperidine. IR spectrum of this product revealed absorption bands for ( $\mathrm{OH}$ groups ) and absence of $\left(\mathrm{NH}_{2}\right)$ and its ${ }^{\mathrm{H}-\mathrm{NMR}}$ spectrum (DMSO- $\left.d_{6}\right)$ exhibited no signal due to the methien proton. In addition, the spectrum showed signals of the protons of the sugar residue, a signal due to $\mathrm{CH}_{3}$ group and those due to the aromatic protons (See Experimental, Scheme 2).

\section{Biological evaluation}

All new compounds were evaluated for their antimicrobial properties. The results were compared with those of well known standards. Compounds 1a,d and 2b,c showed high antibacterial activity against Bacills subitils $\left(G^{-}\right)$. Also, Compound $3 \mathrm{c}$ showed high antifungal activity against Aspergillus niger. Meanwhile, compounds $2 \mathrm{~b}, \mathrm{c}$ and $3 \mathrm{a}$ have moderate (fair) antibacterial activity against Escherichia coli $\left(G^{+}\right)$and $3 \mathrm{a}, 3 \mathrm{~b}$ and $4 \mathrm{c}$ have moderate (fair) antiyeast activity against Candida albicans (yeast).

Also, compound 4a showed high antibacterial activity against Candida albicans (yeast) and moderate (fair) antibacterial activity against other microorganisms when compared to the reference. Meanwhile, compounds $4 \mathrm{~b}$ showed high antibacterial activity against Staphylococcus aureus $\left(G^{+}\right)$. The results of the preliminary Screening fest are listed in (Table 1).

\section{Experimental}

All melting points are uncorrected. Microanalyses were carried out at the Microanalytical Unit, National Research Center and Faculty of Science, Cairo University. The IR spectra (KBr) were recorded on a FT-IR NEXCES Spectrophotometer (Shimadzu, Japan). ${ }^{1} \mathrm{H}-\mathrm{NMR}$ and ${ }^{13} \mathrm{C}-\mathrm{NMR}$ spectra were measured with a Jeol ECA $500 \mathrm{MHz}$ (Japan) in DMSO- $d_{6}$ or $\mathrm{CDCl}_{3}$ and chemical shifts were recorded in $\delta$ ppm relative to TMS. Mass spectra (EI) were run at $70 \mathrm{eV}$ with a Finnigan SSQ 7000 Spectrometer (Thermo-Instrument System Incorporation, USA). All reactions were followed up by TLC. 2-(or 4-) Hydrazinothienopyrimidone derivatives $1 \mathrm{a}, \mathrm{d}$ and $2 \mathrm{~b}, \mathrm{c}$. were prepared according to the literature procedures ${ }^{(14-16)}$. All the physical data are in (Table 2). 
TABLE 1. The antimicrobial activity of the newly synthesized compounds.

\begin{tabular}{|c|c|c|c|c|c|}
\hline \multirow{2}{*}{$\begin{array}{l}\text { Tested } \\
\text { compounds \& } \\
\text { Standers }(\mu \mathrm{g} / \mathrm{ml} \\
\text { lot. Bioanalyse) }\end{array}$} & \multicolumn{5}{|c|}{ Inhibition zone diameter ( $\mathrm{mm}$ / mg sample ) } \\
\hline & $\begin{array}{c}\text { Escherichia } \\
\operatorname{coli}\left(\mathbf{G}^{+}\right)\end{array}$ & $\begin{array}{l}\text { Staphylococus } \\
\text { aureus }\left(\mathbf{G}^{+}\right)\end{array}$ & $\begin{array}{c}\text { Bacills } \\
\text { subitils } \\
\left(\mathbf{G}^{-}\right)\end{array}$ & $\begin{array}{c}\text { Candida } \\
\text { albicans } \\
\text { (yeast) }\end{array}$ & $\begin{array}{c}\text { Aspergillus } \\
\text { niger } \\
\text { (fungi) }\end{array}$ \\
\hline $1 \mathrm{a}, \mathrm{d}$ & + & + & +++ & + & + \\
\hline $2 \mathrm{~b}, \mathrm{c}$ & ++ & + & +++ & + & + \\
\hline $3 \mathrm{a}$ & ++ & + & + & ++ & - \\
\hline $3 \mathrm{~b}$ & + & + & + & ++ & ++ \\
\hline $3 \mathrm{c}$ & - & - & + & + & +++ \\
\hline $4 \mathrm{a}$ & ++ & ++ & ++ & +++ & ++ \\
\hline $4 \mathrm{~b}$ & + & +++ & + & + & ++ \\
\hline $4 c$ & + & - & - & ++ & - \\
\hline Tav. & +++ & +++ & +++ & - & - \\
\hline Nys. & - & - & - & - & + \\
\hline
\end{tabular}

+++ Highly Sensitive (21-25 mm); ++ Fairly Sensitive (16-20 mm); + Slightly Sensitive (15-10 mm); - Not sensitive.

(Tav.) is for Tavanic (anti-gram +ve \& anti-gram -ve )

(Nys.) is for Nystatine (antifungal)

TABLE 2. Physical data for the products 3a-10d.

\begin{tabular}{|c|c|c|c|c|c|c|}
\hline No. & m.p. ${ }^{\circ} \mathrm{C}$ & $\begin{array}{c}\text { Yield } \\
\%\end{array}$ & M.F.(M.wt.) & $\% \mathrm{C}$ & $\% \mathrm{H}$ & $\% \mathbf{N}$ \\
\hline $3 \mathbf{a}$ & $285-287$ & 80 & $\begin{array}{c}\mathrm{C}_{18} \mathrm{H}_{14} \mathrm{~N}_{6} \mathrm{OS}_{2} \\
394.484\end{array}$ & $\begin{array}{l}54.80 \\
54.60\end{array}$ & $\begin{array}{l}3.57 \\
3.50\end{array}$ & $\begin{array}{l}21.30 \\
21.25\end{array}$ \\
\hline $\mathbf{3 b}$ & $240-241$ & 75 & $\begin{array}{c}\mathrm{C}_{19} \mathrm{H}_{16} \mathrm{~N}_{6} \mathrm{~S}_{3} \\
424.578\end{array}$ & $\begin{array}{l}53.75 \\
53.70\end{array}$ & $\begin{array}{l}3.79 \\
3.70\end{array}$ & $\begin{array}{l}19.79 \\
19.75\end{array}$ \\
\hline $3 c$ & $235-236.5$ & 80 & $\begin{array}{c}\mathrm{C}_{15} \mathrm{H}_{14} \mathrm{~N}_{6} \mathrm{~S}_{3} \\
374.518\end{array}$ & $\begin{array}{l}48.10 \\
48.00 \\
\end{array}$ & $\begin{array}{l}3.76 \\
3.70\end{array}$ & $\begin{array}{l}22.44 \\
22.40\end{array}$ \\
\hline 3d & $290-292$ & 75 & $\begin{array}{c}\mathrm{C}_{13} \mathrm{H}_{10} \mathrm{~N}_{6} \mathrm{O}_{3} \mathrm{~S}_{2} \\
362.395\end{array}$ & $\begin{array}{l}43.08 \\
43.00 \\
\end{array}$ & $\begin{array}{l}2.78 \\
2.70\end{array}$ & $\begin{array}{l}23.19 \\
23.15\end{array}$ \\
\hline $4 a$ & $265-261$ & 73 & $\begin{array}{c}\mathrm{C}_{19} \mathrm{H}_{14} \mathrm{~N}_{6} \mathrm{O}_{2} \mathrm{~S}_{2} \\
422.494\end{array}$ & $\begin{array}{l}54.01 \\
54.00 \\
\end{array}$ & $\begin{array}{l}3.34 \\
3.30 \\
\end{array}$ & $\begin{array}{l}19.89 \\
19.85 \\
\end{array}$ \\
\hline $4 b$ & $250-252$ & 70 & $\begin{array}{c}\mathrm{C}_{20} \mathrm{H}_{16} \mathrm{~N}_{6} \mathrm{OS}_{3} \\
452.588\end{array}$ & $\begin{array}{l}53.07 \\
53.00 \\
\end{array}$ & $\begin{array}{l}3.56 \\
3.50\end{array}$ & $\begin{array}{l}18.56 \\
18.45 \\
\end{array}$ \\
\hline $4 c$ & $244-245.5$ & 75 & $\begin{array}{c}\mathrm{C}_{16} \mathrm{H}_{14} \mathrm{~N}_{6} \mathrm{OS}_{3} \\
402.528\end{array}$ & $\begin{array}{l}47.74 \\
47.70\end{array}$ & $\begin{array}{l}3.50 \\
3.45\end{array}$ & $\begin{array}{l}20.87 \\
20.80\end{array}$ \\
\hline $4 d$ & $270-272$ & 65 & $\begin{array}{c}\mathrm{C}_{14} \mathrm{H}_{10} \mathrm{~N}_{6} \mathrm{O}_{4} \mathrm{~S}_{2} \\
390.406\end{array}$ & $\begin{array}{l}43.07 \\
43.00\end{array}$ & $\begin{array}{l}2.58 \\
2.50\end{array}$ & $\begin{array}{l}21.52 \\
21.50\end{array}$ \\
\hline $5 \mathbf{b}$ & $200-201.5$ & 50 & $\begin{array}{c}\mathrm{C}_{20} \mathrm{H}_{15} \mathrm{ClN}_{6} \mathrm{~S}_{3} \\
471.034\end{array}$ & $\begin{array}{l}50.99 \\
50.90 \\
\end{array}$ & $\begin{array}{l}3.21 \\
3.20\end{array}$ & $\begin{array}{l}17.84 \\
17.80\end{array}$ \\
\hline $5 c$ & $205-207$ & 55 & $\begin{array}{c}\mathrm{C}_{16} \mathrm{H}_{13} \mathrm{ClN}_{6} \mathrm{~S}_{3} \\
420.973\end{array}$ & $\begin{array}{l}45.65 \\
45.60\end{array}$ & $\begin{array}{l}3.11 \\
3.09\end{array}$ & $\begin{array}{l}19.96 \\
19.90\end{array}$ \\
\hline $6 a$ & $260-261$ & 80 & $\begin{array}{c}\mathrm{C}_{19} \mathrm{H}_{15} \mathrm{~N}_{7} \mathrm{OS}_{2} \\
421.509\end{array}$ & $\begin{array}{l}54.14 \\
54.10\end{array}$ & $\begin{array}{l}3.58 \\
3.55\end{array}$ & $\begin{array}{l}23.26 \\
23.20\end{array}$ \\
\hline $6 \mathbf{b}$ & $240-242$ & 75 & $\begin{array}{c}\mathrm{C}_{20} \mathrm{H}_{17} \mathrm{~N}_{7} \mathrm{~S}_{3} \\
451.604\end{array}$ & $\begin{array}{l}53.19 \\
53.10\end{array}$ & $\begin{array}{l}3.79 \\
3.70\end{array}$ & $\begin{array}{l}21.71 \\
21.70\end{array}$ \\
\hline $6 c$ & $235-236$ & 80 & $\begin{array}{c}\mathrm{C}_{16} \mathrm{H}_{15} \mathrm{~N}_{7} \mathrm{~S}_{3} \\
401.543\end{array}$ & $\begin{array}{l}47.86 \\
47.80\end{array}$ & $\begin{array}{l}3.76 \\
3.70\end{array}$ & $\begin{array}{l}24.41 \\
24.40\end{array}$ \\
\hline 6d & $265-266.5$ & 75 & $\begin{array}{c}\mathrm{C}_{14} \mathrm{H}_{11} \mathrm{~N}_{7} \mathrm{O}_{3} \mathrm{~S}_{2} \\
389.421\end{array}$ & $\begin{array}{l}43.18 \\
43.16\end{array}$ & $\begin{array}{l}2.84 \\
2.82\end{array}$ & $\begin{array}{l}25.17 \\
25.15\end{array}$ \\
\hline $7 a$ & $250-252$ & 78 & $\begin{array}{c}\mathrm{C}_{19} \mathrm{H}_{22} \mathrm{~N}_{4} \mathrm{O}_{6} \mathrm{~S} \\
434.475\end{array}$ & $\begin{array}{l}52.52 \\
52.50\end{array}$ & $\begin{array}{l}5.10 \\
5.08\end{array}$ & $\begin{array}{l}12.89 \\
12.85\end{array}$ \\
\hline
\end{tabular}

Egypt. J. Chem. 53, No. 4 (2010) 
TABLE 2. Cont.

\begin{tabular}{|c|c|c|c|c|c|c|}
\hline No. & m.p. ${ }^{\circ} \mathrm{C}$ & $\begin{array}{c}\text { Yield } \\
\%\end{array}$ & M.F.(M.wt.) & $\% \mathrm{C}$ & $\% \mathrm{H}$ & $\% \mathbf{N}$ \\
\hline $7 \mathbf{b}$ & $245-247$ & 80 & $\begin{array}{c}\mathrm{C}_{19} \mathrm{H}_{22} \mathrm{~N}_{4} \mathrm{O}_{6} \mathrm{~S} \\
434.475\end{array}$ & $\begin{array}{l}52.52 \\
52.52\end{array}$ & $\begin{array}{l}5.10 \\
5.07\end{array}$ & $\begin{array}{l}12.89 \\
12.86\end{array}$ \\
\hline $7 c$ & $233-234.5$ & 73 & $\begin{array}{c}\mathrm{C}_{18} \mathrm{H}_{20} \mathrm{~N}_{4} \mathrm{O}_{5} \mathrm{~S} \\
404.449\end{array}$ & $\begin{array}{l}53.45 \\
53.44\end{array}$ & $\begin{array}{l}4.98 \\
4.95\end{array}$ & $\begin{array}{l}13.85 \\
13.83\end{array}$ \\
\hline $9 \mathbf{a}$ & $153-154$ & 70 & $\begin{array}{c}\mathrm{C}_{29} \mathrm{H}_{30} \mathrm{~N}_{4} \mathrm{O}_{11} \mathrm{~S} \\
642.63\end{array}$ & $\begin{array}{l}54.20 \\
54.18\end{array}$ & $\begin{array}{l}4.71 \\
4.70\end{array}$ & $\begin{array}{l}8.72 \\
8.70\end{array}$ \\
\hline $9 \mathrm{~b}$ & $145-146$ & 73 & $\begin{array}{c}\mathrm{C}_{29} \mathrm{H}_{30} \mathrm{~N}_{4} \mathrm{O}_{11} \mathrm{~S} \\
642.63\end{array}$ & $\begin{array}{l}54.20 \\
54.19\end{array}$ & $\begin{array}{l}4.71 \\
4.71\end{array}$ & $\begin{array}{l}8.72 \\
8.70\end{array}$ \\
\hline $9 \mathrm{c}$ & $140-142$ & 70 & $\begin{array}{c}\mathrm{C}_{26} \mathrm{H}_{26} \mathrm{~N}_{4} \mathrm{O}_{9} \mathrm{~S} \\
570.57\end{array}$ & $\begin{array}{l}54.73 \\
54.72\end{array}$ & $\begin{array}{l}4.59 \\
4.57\end{array}$ & $\begin{array}{l}9.82 \\
9.80\end{array}$ \\
\hline 10a & $245-247$ & 51 & $\begin{array}{c}\mathrm{C}_{19} \mathrm{H}_{20} \mathrm{~N}_{4} \mathrm{O}_{6} \mathrm{~S} \\
432.448\end{array}$ & $\begin{array}{l}52.77 \\
52.75\end{array}$ & $\begin{array}{l}4.66 \\
4.65\end{array}$ & $\begin{array}{l}12.96 \\
12.95\end{array}$ \\
\hline 10b & $259-260$ & 50 & $\begin{array}{c}\mathrm{C}_{18} \mathrm{H}_{20} \mathrm{~N}_{4} \mathrm{O}_{6} \mathrm{~S} \\
420.448\end{array}$ & $\begin{array}{l}52.77 \\
52.73\end{array}$ & $\begin{array}{l}4.66 \\
4.64\end{array}$ & $\begin{array}{l}12.96 \\
12.93\end{array}$ \\
\hline $10 \mathrm{c}$ & $250-251.5$ & 48 & $\begin{array}{c}\mathrm{C}_{18} \mathrm{H}_{18} \mathrm{~N}_{4} \mathrm{O}_{5} \mathrm{~S} \\
402.422\end{array}$ & $\begin{array}{l}53.72 \\
53.71\end{array}$ & $\begin{array}{l}4.51 \\
4.51\end{array}$ & $\begin{array}{l}13.92 \\
13.91\end{array}$ \\
\hline 11 & $260-262$ & 60 & $\begin{array}{c}\text { C19H20N4O6S } \\
432.448\end{array}$ & $\begin{array}{l}52.77 \\
52.76\end{array}$ & $\begin{array}{l}4.66 \\
4.63\end{array}$ & $\begin{array}{l}12.96 \\
12.96\end{array}$ \\
\hline
\end{tabular}

Preparation of compounds $3 a-d$

General method

Compound 1a,d or $2 \mathrm{~b}, \mathrm{c}$ (0.01 mole) was refluxed with [bis(methylthio) methylene] malononitrile ( 0.01 mole) in methanol, for five hours. The reaction mixture was allowed to cool to room temperature. The precipitate so-formed was collected by filtration and recrystallized from proper solvent to produce $3 \mathrm{a}-\mathrm{d}$.

5-Amino-1-(5-methyl-4-oxo-6-phenyl-3,4-dihydrothieno[2,3-d] pyramidine-2-yl)3-(methylthio)-1H-pyrazole-4-carbonitrile (3a)

Compound $3 \mathrm{a}$ was obtained by reacting of $1 \mathrm{a}(2.72 \mathrm{~g}, 0.01$ mole) with [bis(methylthio)methylene]malononitrile $(1.70 \mathrm{~g}, 0.01$ mole). The product was recrystallized from dioxane to produce $3 \mathrm{a}$ as colorless crystals. IR spectrum $\left(\mathrm{KBr}, \mathrm{cm}^{-1}\right): 3365(\mathrm{NH}) ; 3293,3203\left(\mathrm{NH}_{2}\right) ; 2228(\mathrm{CN})$ and $1686(\mathrm{CO}) ;{ }^{1} \mathrm{H}-\mathrm{NMR}$ (DMSO- $\left.d_{6}\right) \delta$ ppm: $2.54\left(\mathrm{~s}, 3 \mathrm{H}, \mathrm{CH}_{3}\right), 2.66\left(\mathrm{~s}, 3 \mathrm{H}, \mathrm{SCH}_{3}\right), 7.51-7.60$ (m, 5H, Ar$\mathrm{H}), 6.31\left(\mathrm{~s}, 2 \mathrm{H}, \mathrm{NH}_{2}, \mathrm{D}_{2} \mathrm{O}\right.$ exchangeable $)$ and $9.02\left(\mathrm{~s}, 1 \mathrm{H}, \mathrm{NH}, \mathrm{D}_{2} \mathrm{O}-\right.$ exchangeable); ${ }^{13} \mathrm{C}-\mathrm{NMR}$ (DMSO- $\left.d_{6}\right) \delta$ ppm: 9.30 $\left(\mathrm{CH}_{3}\right), 12.08 \quad\left(\mathrm{SCH}_{3}\right)$, 117.1(CN), 76.1, 142.3, 148.4-pyrazol ring carbon atoms), 126.4-128.7 ( $\mathrm{Ar}-$ carbon atoms), 133.7-158.0 (thienopyrimidone carbon atoms) and 161.1 (CO); MS (m/z): 394(60\%).

5-Amino-1- (5-methyl-2- (methylthio) -6-phenylthieno [2,3-d] pyramidin-4-yl) -3(methylthio)-1H-pyrazole-4-carbonitrile (3b)

Compound $3 \mathrm{~b}$ was obtained by reacting of $2 \mathrm{~b}(3.02 \mathrm{~g}, 0.01$ mole) with [bis(methylthio)methylene]malononitrile $(1.70 \mathrm{~g}, 0.01$ mole). The product was recrystallized from dioxane to produce $3 \mathrm{~b}$ as colorless crystals. IR spectrum $\left(\mathrm{KBr}, \mathrm{cm}^{-1}\right): 3306,3232\left(\mathrm{NH}_{2}\right)$ and $2224(\mathrm{CN}) ;{ }^{1} \mathrm{H}-\mathrm{NMR}$ (DMSO- $\left.d_{6}\right) \delta$ ppm: 2.56 
(s, 3H, $\left.\mathrm{CH}_{3}\right), 2.60\left(\mathrm{~s}, 3 \mathrm{H}, \mathrm{SCH}_{3}\right), 2.62\left(\mathrm{~s}, 3 \mathrm{H}, \mathrm{SCH}_{3}\right) 6.29\left(\mathrm{~s}, 2 \mathrm{H}, \mathrm{NH}_{2}, \mathrm{D}_{2} \mathrm{O}-\right.$ exchangeable) and 7.51-7.60 (m, 5H, Ar-H); MS (m/z): 424(100\%).

5-Amino-1-(2-methylthiocyclopenta[4,5] thieno[2,3-d]pyrimidin-4-yl)-3-(methylthio) -1H-pyrazole-4-carbonitrile (3c)

Compound $3 \mathrm{c}$ was obtained by reacting of $2 \mathrm{c}(2.52 \mathrm{~g}, 0.01$ mole $)$ with [bis(methylthio)methylene]malononitrile $(1.70 \mathrm{~g}, 0.01$ mole). The product was recrystallized from dioxane to produce $3 \mathrm{c}$ as colorless crystals. IR spectrum $\left(\mathrm{KBr}, \mathrm{cm}^{-1}\right)$ : 3264, $3221\left(\mathrm{NH}_{2}\right)$ and $2225(\mathrm{CN})$; ${ }^{1} \mathrm{H}-\mathrm{NMR}$ (DMSO- $\left.d_{6}\right) \mathrm{d} \mathrm{ppm:}$ $2.45\left(\mathrm{~m}, 2 \mathrm{H}, \mathrm{CH}_{2}\right), 2.80\left(\mathrm{~m}, 4 \mathrm{H}, 2 \mathrm{CH}_{2}\right), 3.35\left(\mathrm{~s}, 3 \mathrm{H}, \mathrm{SCH}_{3}\right), 3.70\left(\mathrm{~s}, 3 \mathrm{H}, \mathrm{SCH}_{3}\right)$ and $6.50\left(\mathrm{~s}, 2 \mathrm{H}, \mathrm{NH}_{2}, \mathrm{D}_{2} \mathrm{O}\right.$-exchangeable); $\mathrm{MS}(\mathrm{m} / \mathrm{z}): 374(75 \%)$.

2-(5-Amino-4-cyano-3-(methylthio)-1H-pyrazol-1-yl)-5-methyl-4-oxo-3,4-dihydrothieno[2,3-d]pyrimidine-6-carboxylic acid (3d)

Compound $3 \mathrm{~d}$ was obtained by reacting of $1 \mathrm{~d}(2.40 \mathrm{~g}, 0.01$ mole) with [bis(methylthio)methylene]malononitrile $(1.70 \mathrm{~g}, 0.01$ mole). The product was recrystallized from dioxane to produce $3 \mathrm{~d}$ as colorless crystals. IR spectrum $\left(\mathrm{KBr}, \mathrm{cm}^{-1}\right): 3394(\mathrm{OH}), 3345-3216\left[\left(\mathrm{NH}_{2}\right),(\mathrm{NH})\right], 2210(\mathrm{CN}), 1700(\mathrm{CO}$ carboxylic) and $1668\left(\mathrm{CO}\right.$, amide); ${ }^{1} \mathrm{H}-\mathrm{NMR}\left(\mathrm{DMSO}-d_{6}\right) \delta \mathrm{ppm}: 2.44(\mathrm{~s}, 3 \mathrm{H}$, $\mathrm{CH}_{3}$ ), 2.64 (s, 3H, $\mathrm{SCH}_{3}$ ), 6.40 (s, 2H, $\mathrm{NH}_{2}, \mathrm{D}_{2} \mathrm{O}$-exchangeable), 7.04 (s, 1H, NH, $\mathrm{D}_{2} \mathrm{O}$ exchangeable), and 12.02 (br.s, $1 \mathrm{H}, \mathrm{OH}, \mathrm{D}_{2} \mathrm{O}$ exchangeable); $\mathrm{MS}(\mathrm{m} / \mathrm{z})$ : $362(65 \%)$.

\section{Preparation of compounds $4 a-d$}

General method

A mixture of $3 \mathrm{a}-\mathrm{d}(0.01 \mathrm{~mole})$, formic acid $(10 \mathrm{ml})$ and a catalytic amount of concentrated hydrochloric acid was heated under reflux for $10 \mathrm{hr}$. The reaction mixture was allowed to cool to room temperature. The precipitate so-formed was collected by filtration and recrystallized from ethanol to produce $4 \mathrm{a}-\mathrm{d}$.

5-Methyl-2-(3-(methylthio)-4-oxo-4,5-dihydro-1H-pyrazolo[3,4-d] pyrimidin-1yl)-6-phenylthieno[2,3-d]pyrimidin-4(3H)-one (4a)

Compound $4 \mathrm{a}$ was obtained by reacting of $3 \mathrm{a}(3.94 \mathrm{~g}, 0.01 \mathrm{~mole})$ and formic acid $(10 \mathrm{ml})$ as white crystals. IR spectrum $\left(\mathrm{KBr}, \mathrm{cm}^{-1}\right): 3446($ broad $\mathrm{OH})$, 3112(NH) and 1673(CO); ${ }^{1} \mathrm{H}-\mathrm{NMR}\left(\mathrm{DMSO}-d_{6}\right) \delta \mathrm{ppm}: 2.58\left(\mathrm{~s}, 3 \mathrm{H}, \mathrm{CH}_{3}\right), 2.70$ $\left(\mathrm{s}, 3 \mathrm{H}, \mathrm{SCH}_{3}\right), 7.50-7.58(\mathrm{~m}, 5 \mathrm{H}, \mathrm{Ar}-\mathrm{H}), 7.70(\mathrm{~s}, 1 \mathrm{H}, \mathrm{CH}), 9.02\left(\mathrm{~s}, 1 \mathrm{H}, \mathrm{NH}, \mathrm{D}_{2} \mathrm{O}-\right.$ exchangeable) and $12.10\left(\mathrm{~s}, 1 \mathrm{H}, \mathrm{OH}, \mathrm{D}_{2} \mathrm{O}\right.$ exchangeable); $\mathrm{MS}(\mathrm{m} / \mathrm{z}): 422(50 \%)$.

1-(5-Methyl-2-(methylthio)-6-phenylthieno[2,3-d] pyrimidin-4-yl)3-(methyllthio)$1 \mathrm{H}$-pyrazolo[3,4-d]pyrimidin-4(5H)-one $(4 b)$

Compound $4 \mathrm{~b}$ was obtained by reacting of $3 \mathrm{~b}(4.24 \mathrm{~g}, 0.01 \mathrm{~mole})$ and formic acid $(10 \mathrm{ml})$ as white crystals. IR spectrum $\left(\mathrm{KBr}, \mathrm{cm}^{-1}\right): 3430(\mathrm{OH}) ;{ }^{1} \mathrm{H}-\mathrm{NMR}$ $\left(\mathrm{DMSO}-d_{6}\right) \delta \mathrm{ppm}: 2.54\left(\mathrm{~s}, 3 \mathrm{H}, \mathrm{CH}_{3}\right), 2.59\left(\mathrm{~s}, 3 \mathrm{H}, \mathrm{SCH}_{3}\right), 2.65\left(\mathrm{~s}, 3 \mathrm{H}, \mathrm{SCH}_{3}\right)$, 7.50-7.54 (m, 5H, Ar-H), 8.00(s, $1 \mathrm{H}, \mathrm{CH})$ and $11.30\left(\mathrm{~s}, 1 \mathrm{H}, \mathrm{OH}, \mathrm{D}_{2} \mathrm{O}-\right.$ exchangeable); MS (m/z): 452 (65\%). 
1-(2-Methylthiocyclopenta[4,5]thieno[2,3-d]pyrimidin-4-yl)-3- (methyl-thio)-1Hpyrazolo[3,4-d]pyrimidin-4(5H)-one (4c)

Compound $4 \mathrm{c}$ was obtained by reacting of $3 \mathrm{c}(3.74 \mathrm{~g}, 0.01 \mathrm{~mole})$ and formic acid $(10 \mathrm{ml})$ as white crystals. IR spectrum $\left(\mathrm{KBr}, \mathrm{cm}^{-1}\right): 3500(\mathrm{OH}) ;{ }^{1} \mathrm{H}-\mathrm{NMR}$ $\left(\mathrm{DMSO}-d_{6}\right) \delta \mathrm{ppm}: 2.47\left(\mathrm{~m}, 2 \mathrm{H}, \mathrm{CH}_{2}\right), 2.55\left(\mathrm{~s}, 3 \mathrm{H}, \mathrm{SCH}_{3}\right), 2.57\left(\mathrm{~s}, 3 \mathrm{H}, \mathrm{SCH}_{3}\right)$, $2.83\left(\mathrm{~m}, 2 \mathrm{H}, \mathrm{CH}_{2}\right), 2.85\left(\mathrm{~m}, 2 \mathrm{H}, \mathrm{CH}_{2}\right)$ and 8.01(s, 1H, CH); MS (m/z): 402 $(75 \%)$.

5-Methyl-2-(3-(methylthio)-4-oxo-4,5-dihydro-1H-pyrazolo[3,4-d]pyri-midine-1yl)-4-oxo-3,4-dihydrothieno[2,3-d]pyrimidine-6- carboxylic acid (4d)

Compound $4 \mathrm{~d}$ was obtained by reacting of $3 \mathrm{~d}(3.95 \mathrm{~g}, 0.01 \mathrm{~mole})$ and formic acid $(10 \mathrm{ml})$ as white crystals. IR spectrum $\left(\mathrm{KBr}, \mathrm{cm}^{-1}\right): 3445,3330(2 \mathrm{OH}), 3210$ $(\mathrm{NH})$, and 1720,1668(2CO); ${ }^{1} \mathrm{H}-\mathrm{NMR}$ (DMSO- $\left.d_{6}\right) \delta \mathrm{ppm}: 2.44\left(\mathrm{~s}, 3 \mathrm{H}, \mathrm{CH}_{3}\right)$, $2.70\left(\mathrm{~s}, 3 \mathrm{H}, \mathrm{SCH}_{3}\right), 7.95(\mathrm{~s}, 1 \mathrm{H}, \mathrm{CH}), 8.40\left(\mathrm{~s}, 1 \mathrm{H}, \mathrm{NH}, \mathrm{D}_{2} \mathrm{O}\right.$ exchangeable $), 11.10$ (s, 1H, OH, $\mathrm{D}_{2} \mathrm{O}$ exchangeable), and 12.20 (br.s, 1H, OH, $\mathrm{D}_{2} \mathrm{O}$ exchangeable); MS (m/z): $390(80 \%)$.

Preparation of compounds $5 b$ or $5 c$

General method

A mixture of compound $4 \mathrm{~b}$ or $4 \mathrm{c}(0.01$ mole $)$ in dry dioxane $(30 \mathrm{ml})$ and phosphorus oxychloride $(7 \mathrm{ml})$ was stirred under reflux for $3 \mathrm{hr}$. The reaction mixture was allowed to cool to room temperature and poured onto ice-water $(150 \mathrm{~g})$. The precipitate so-formed was collected by filtration and recrystallized from benzene to produce $5 \mathrm{~b}$ or $5 \mathrm{c}$ as white crystals.

4- (4-Chloro-3- (methylthio) -1H-pyrazolo [3,4-d] pyrimidin-1-yl) -5-meth-yl-2(methylthio) -6-phenylthieno[2,3-d]pyrimidine) (5b)

Compound $5 \mathrm{~b}$ was obtained by reacting of $4 \mathrm{~b}(4.52 \mathrm{~g}, 0.01 \mathrm{~mole})$ and phosphorus oxychloride $(7 \mathrm{ml})$ as white crystals. ${ }^{1} \mathrm{H}-\mathrm{NMR}\left(\mathrm{DMSO}-d_{6}\right) \delta \mathrm{ppm}$ : $2.56\left(\mathrm{~s}, 3 \mathrm{H}, \mathrm{CH}_{3}\right), 2.59\left(\mathrm{~s}, 3 \mathrm{H}, \mathrm{SCH}_{3}\right), 2.63\left(\mathrm{~s}, 3 \mathrm{H}, \mathrm{SCH}_{3}\right), 7.50-7.54(\mathrm{~m}, 5 \mathrm{H}, \mathrm{Ar}-$ $\mathrm{H}), 8.00(\mathrm{~s}, 1 \mathrm{H}, \mathrm{CH})$; MS (m/z): 470 and $472\left[\mathrm{M}^{+},(100 \%, 46.6 \%)\right]$.

4-(4-Chloro-3-(methylthio)-1H-pyrazolo[3,4-d]pyrimidin-1-yl)-2-methy-lthiocyclopenta[4,5]thieno[2,3-d]pyrimidin (5c)

Compound $5 \mathrm{c}$ was obtained by reacting of $4 \mathrm{c}(4.02 \mathrm{~g}, 0.01 \mathrm{~mole})$ and phosphorus oxychloride $(7 \mathrm{ml})$ as white crystals. ${ }^{1} \mathrm{H}-\mathrm{NMR}$ (DMSO- $\left.d_{6}\right) \delta \mathrm{ppm}$ : 2.44(m, $\left.2 \mathrm{H}, \mathrm{CH}_{2}\right), 2.56\left(\mathrm{~s}, 3 \mathrm{H}, \mathrm{SCH}_{3}\right), 2.60$ (s, 3H, SCH$), 2.80\left(\mathrm{~m}, 2 \mathrm{H}, \mathrm{CH}_{2}\right)$, $2.85\left(\mathrm{~m}, 2 \mathrm{H}, \mathrm{CH}_{2}\right)$ and $8.10(\mathrm{~s}, 1 \mathrm{H}, \mathrm{CH}) ; \mathrm{MS}(\mathrm{m} / \mathrm{z}): 420$ and $422\left[\mathrm{M}^{+},(82 \%, 25.5\right.$ $\%)]$.

Preparation of compounds $6 a-d$

General method

A mixture of $3 \mathrm{a}-\mathrm{d}(0.01 \mathrm{~mole})$ and formamide $(10 \mathrm{ml})$ was stirred under reflux in dimethylformamide for $6 \mathrm{hr}$. The reaction mixture was allowed to cool to room temperature and poured onto water $(150 \mathrm{ml})$. The precipitate so-formed was collected by filtration and recrystallized from dioxane to produce $6 \mathrm{a}-\mathrm{d}$.

Egypt. J. Chem. 53, No. 4 (2010) 
2-(4-Amino-3- (methylthio) -1H-pyrazolo [3,4-d] pyrimidine-1-yl) -5-methyl-6phenylthieno[2,3-d]pyrimidine-4(3H)-one (6a)

Compound 6 a was obtained by reacting $3 \mathrm{a}(3.94 \mathrm{~g}, 0.01 \mathrm{~mole})$ with formamide $(10 \mathrm{ml})$ as brown crystals. IR spectrum $\left(\mathrm{KBr}, \mathrm{cm}^{-1}\right): 3320,3307\left(\mathrm{NH}_{2}\right), 3200$ $(\mathrm{NH})$ and $1666(\mathrm{CO}) ;{ }^{1} \mathrm{H}-\mathrm{NMR}\left(\mathrm{DMSO}-d_{6}\right) \delta \mathrm{ppm}: 2.58\left(\mathrm{~s}, 3 \mathrm{H}, \mathrm{CH}_{3}\right), 2.75(\mathrm{~s}$, $3 \mathrm{H}, \mathrm{SCH}_{3}$ ), 4.9 (br.s, $2 \mathrm{H}, \mathrm{NH}_{2}, \mathrm{D}_{2} \mathrm{O}$ exchangeable), 7.51-7.58 (m, 5H,Ar-H), 7.74 (s, 1H, CH) and 9.02 (br.s, 1H, NH, $\mathrm{D}_{2} \mathrm{O}$ exchangeable); $\mathrm{MS}(\mathrm{m} / \mathrm{z}): 421$ (95\%).

1-(5-Methyl-2- (methylthio) -6-phenylthieno [2,3-d] pyrimidine-4-yl) -4-amino-3(methylthio)-1H-pyrazolo[3,4-d]pyrimidine (6b)

Compound $6 \mathrm{~b}$ was obtained by reacting $3 \mathrm{~b}(4.24 \mathrm{~g}, 0.01 \mathrm{~mole})$ with formamide $(10 \mathrm{ml})$ as yellow crystals. IR spectrum $\left(\mathrm{KBr}, \mathrm{cm}^{-1}\right): 3320,3311\left(\mathrm{NH}_{2}\right) ;{ }^{1} \mathrm{H}-\mathrm{NMR}$ $\left(\mathrm{DMSO}-d_{6}\right) \delta \mathrm{ppm}: 2.53\left(\mathrm{~s}, 3 \mathrm{H}, \mathrm{CH}_{3}\right), 2.57\left(\mathrm{~s}, 3 \mathrm{H}, \mathrm{SCH}_{3}\right), 2.60\left(\mathrm{~s}, 3 \mathrm{H}, \mathrm{SCH}_{3}\right), 5.2$ (br.s, $2 \mathrm{H}, \mathrm{NH}_{2}, \mathrm{D}_{2} \mathrm{O}$ exchangeable), and 7.50-7.54 (m, 5H, Ar-H), 8.00(s, $1 \mathrm{H}$, $\mathrm{CH}) ; \mathrm{MS}(\mathrm{m} / \mathrm{z}): 451$ (90\%).

1-(2-Methylthiocyclopenta[4,5]thieno[2,3-d]pyrimidin-4-yl)-4-amino-3-(methylthio)1H-pyrazolo[3,4-d]pyrimidine (6c)

Compound $6 \mathrm{c}$ was obtained by reacting $3 \mathrm{c}(3.74 \mathrm{~g}, 0.01 \mathrm{~mole})$ with formamide $(10 \mathrm{ml})$ as dark yellow crystals. IR spectrum $\left(\mathrm{KBr}, \mathrm{cm}^{-1}\right): 3326,3315\left(\mathrm{NH}_{2}\right) ;{ }^{1} \mathrm{H}-$ NMR (DMSO- $\left.d_{6}\right) \delta$ ppm: $2.40\left(\mathrm{~m}, 2 \mathrm{H}, \mathrm{CH}_{2}\right), 2.52\left(\mathrm{~s}, 3 \mathrm{H}, \mathrm{SCH}_{3}\right), 2.60(\mathrm{~s}$, $\left.3 \mathrm{H}, \mathrm{SCH}_{3}\right), 2.80\left(\mathrm{~m}, 2 \mathrm{H}, \mathrm{CH}_{2}\right), 2.85\left(\mathrm{~m}, 2 \mathrm{H}, \mathrm{CH}_{2}\right), 6.0$ (br.s, $2 \mathrm{H}, \mathrm{NH}_{2}, \mathrm{D}_{2} \mathrm{O}$ exchangeable) and 8.01(s, 1H, CH); MS (m/z): 401 (79\%)

2-(4-Amino-3-(methylthio) -1H-pyrazolo[3,4-d]pyrimidine-1-yl)-5-methyl-4-oxo3 ,4-dihydrothieno[2, 3-d]pyrimidine-6-Carboxylic acid (6d)

Compound $6 \mathrm{~d}$ was obtained by reacting $3 \mathrm{~d}(3.95 \mathrm{~g}, 0.01 \mathrm{~mole})$ with formamide $(10 \mathrm{ml})$ as white crystals. IR spectrum $\left(\mathrm{KBr}, \mathrm{cm}^{-1}\right): 3354$ (broad $\left.\mathrm{OH}\right)$, 3333(NH),3317,3310 $\left(\mathrm{NH}_{2}\right)$ and 1671,1666(2CO). ${ }^{1} \mathrm{H}-\mathrm{NMR}$ (DMSO- $\left.d_{6}\right) \delta \mathrm{ppm}$ : 2.40 (s, 3H, $\mathrm{CH}_{3}$ ), 2.65 (s, 3H, $\mathrm{SCH}_{3}$ ), 5.8 (br.s, $2 \mathrm{H}, \mathrm{NH}_{2}, \mathrm{D}_{2} \mathrm{O}$ exchangeable), 7.10 (br.s, $1 \mathrm{H}, \mathrm{NH}, \mathrm{D}_{2} \mathrm{O}$ exchangeable), $8.02(\mathrm{~s}, 1 \mathrm{H}, \mathrm{CH})$ and 10.95 (br.s, $1 \mathrm{H}$, $\mathrm{OH}, \mathrm{D}_{2} \mathrm{O}$ exchangeable); MS (m/z): 389 (100\%).

\section{Preparation of compounds $7 a-c$}

General method

A mixture of compound 1a (0.01 mole), monosaccharide (aldoses), namely, D-glucose $(1.80 \mathrm{~g}, 0.01$ mole) or D-mannose $(1.80 \mathrm{~g}, 0.01 \mathrm{~mole})$, or D-arabinose $(1.50 \mathrm{~g}, 0.01 \mathrm{~mole})$ in boiling dioxane in the presence of catalytic amounts of piperidine was stirred under reflux for $5 \mathrm{hr}$. The precipitate so-formed was collected by filtration and recrystallized from dioxane to produce $7 \mathrm{a}-\mathrm{c}$.

2-Glucosylhydrazino-5-methyl-6-phenylthieno[2,3-d]pyrimidine-4(4H)-one (7a)

Compound $7 \mathrm{a}$ was obtained by reaction of $1 \mathrm{a}(2.72 \mathrm{~g}, 0.01 \mathrm{~mole})$ and $\mathrm{D}$ glucose $\left(1.80 \mathrm{~g}, 0.01\right.$ mole) as deep yellow crystals, IR spectrum $\left(\mathrm{KBr}, \mathrm{cm}^{-1}\right)$ : $3200(\mathrm{NH})$ and $1675(\mathrm{CO}) .{ }^{1} \mathrm{H}-\mathrm{NMR}\left(\mathrm{DMSO}-d_{6}\right) \delta \mathrm{ppm}: 2.82\left(\mathrm{~s}, 3 \mathrm{H}, \mathrm{CH}_{3}\right), 3.55$ $3.65\left(\mathrm{~m}, 5 \mathrm{H}, 5 \mathrm{OH}, \mathrm{D}_{2} \mathrm{O}\right.$ exchangeable), $3.81\left(\mathrm{~m}, 2 \mathrm{H}, \mathrm{CH}_{2} \mathrm{OH}\right), 4.35(\mathrm{~m}, 1 \mathrm{H}, \mathrm{H}-$ 4), 4.50 (m, 1H, H-2`), 4.65 (d, J=6.65Hz,1H, H-3`), 5.50 (d, J=7.25Hz,1H, H- 
1) $)$ 7.40-7.50 (m, 5H, Ar-H), $7.55(\mathrm{~s}, 1 \mathrm{H}, \mathrm{CH}), 11.30$ (br.s, 1H, NH, D 2 exchangeable) and 10.55 (br.s, $1 \mathrm{H}, \mathrm{NH}, \mathrm{D}_{2} \mathrm{O}$ exchangeable); ${ }^{13} \mathrm{C}-\mathrm{NMR}$ (DMSO$\left.d_{6}\right) \delta$ ppm: 9.30 $\left(\mathrm{CH}_{3}\right), 64.4\left(\mathrm{CH}_{2}\right), 66.8$ - $72.3(4 \mathrm{CH}), 126.2$ - 133.7 (Ar-C), 135.5158.0 (thienopyrimidone carbon atoms and glucose $\mathrm{C}-1$ carbon atom) and 163.1 (CO); MS (m/z): 434 (85\%).

2-Mannosylhydrazino-5-methyl-6-phenylthieno[2,3-d]pyrimidine-4(4H)-one (7b)

Compound $7 \mathrm{~b}$ was obtained by reaction of $1 \mathrm{a}(2.72 \mathrm{~g}, 0.01 \mathrm{~mole})$ and $\mathrm{D}$ mannose $\left(1.80 \mathrm{~g}, 0.01\right.$ mole) as yellow crystals. IR spectrum $\left(\mathrm{KBr}, \mathrm{cm}^{-1}\right): 3220$ $(\mathrm{NH})$ and $1681(\mathrm{CO})),{ }^{1} \mathrm{H}-\mathrm{NMR}\left(\mathrm{DMSO}-d_{6}\right) \delta \mathrm{ppm}: 2.80\left(\mathrm{~s}, 3 \mathrm{H}, \mathrm{CH}_{3}\right), 3.60-3.65$ (m, 5H, $5 \mathrm{OH}, \mathrm{D}_{2} \mathrm{O}$-exchangeable), $3.90\left(\mathrm{~m}, 2 \mathrm{H}, \mathrm{CH}_{2} \mathrm{OH}\right), 4.29\left(\mathrm{~m}, 1 \mathrm{H}, \mathrm{H}-4^{\prime}\right)$, 4.40 (m, 1H, H-2`), 4.65 (d, J=8.25Hz, 1H, H-3`), 4.90 (d, J=4.75Hz,1H, H-1`), 7.40-7.45 (m, 5H,Ar-H), $7.50(\mathrm{~s}, 1 \mathrm{H}, \mathrm{CH}), 10.30$ (br.s, $1 \mathrm{H}, \mathrm{NH}, \mathrm{D}_{2} \mathrm{O}-$ exchangeable) and 10.53 (br.s, $1 \mathrm{H}, \mathrm{NH}, \mathrm{D}_{2} \mathrm{O}$ exchangeable); MS (m/z): 434 $(90 \%)$.

2-Arabinosylhydrazino-5-methyl-6-phenylthieno[2,3-d]pyrimidine-4(4H)-one (7c)

Compound $7 \mathrm{c}$ was obtained by reaction of $1 \mathrm{a}(2.72 \mathrm{~g}, 0.01 \mathrm{~mole})$ and $\mathrm{D}$ arabinose $\left(1.50 \mathrm{~g}, 0.01\right.$ mole) as deep yellow crystals. IR spectrum $\left(\mathrm{KBr}, \mathrm{cm}^{-1}\right)$ : $3215(\mathrm{NH})$ and $1665(\mathrm{CO}),{ }^{1} \mathrm{H}-\mathrm{NMR}\left(\mathrm{DMSO}-d_{6}\right) \delta \mathrm{ppm}: 2.02\left(\mathrm{~s}, 3 \mathrm{H}, \mathrm{CH}_{3}\right), 3.58$ $3.65\left(\mathrm{~m}, 4 \mathrm{H}, 4 \mathrm{OH}, \mathrm{D}_{2} \mathrm{O}\right.$-exchangeable), $3.85\left(\mathrm{~m}, 2 \mathrm{H}, \mathrm{CH}_{2} \mathrm{OH}\right), 4.25(\mathrm{~m}, 1 \mathrm{H}, \mathrm{H}-$ 3`), 4.40 (m, 1H, H-2`), 4.90 (d, 1H, H-1`), , 7.40-7.50 (m, 5H,Ar-H), 7.50 (s, $1 \mathrm{H}, \mathrm{CH}$ ), 10.35 (br.s, $1 \mathrm{H}, \mathrm{NH}, \mathrm{D}_{2} \mathrm{O}$ exchangeable) and 10.55 (br.s, $1 \mathrm{H}, \mathrm{NH}, \mathrm{D}_{2} \mathrm{O}$ exchangeable); MS (m/z): 404 (70\%).

3-(0-Acetylglycosyl)-6-methyl-5-oxo-7-phenylthieno[2,3-d][1,2,4] triazolo[4,3a]pyrimidine-3-yl $(9 a-c)$

General method

A solution of compound $7 \mathrm{a}-\mathrm{c}(0.01 \mathrm{~mole})$ in a mixture of acetic anhydridepyridine (20ml:20ml) was stirred at room temperature for $7 \mathrm{hr}$. The reaction mixture was poured onto ice-water under stirring then extracted with chloroform several times and after removal of chloroform under reduced pressure, the formed crystals were recrystallized from methanol to produce $9 \mathrm{a}-\mathrm{c}$.

3- (1 ‘2`,3, 4, `5-0- Pentaacetylglycosyl) -6-methyl -5-oxo -7-phenyl-1,5-dihydrothieno [2,3-d] [1,2,4]triazolo[4,3-a]pyrimidine (9a)

A solution of compound $7 \mathrm{a}(4.34 \mathrm{~g}, 0.01 \mathrm{~mole})$ in a mixture of acetic anhydride-pyridine was stirred to produce $9 \mathrm{a}$, as deep yellow crystals. IR spectrum $\left(\mathrm{KBr}, . \mathrm{cm}^{-1}\right): 3200(\mathrm{NH}), 1751-1740(\mathrm{OAc})$ and $1680\left(\mathrm{CO}\right.$ amide), ${ }^{1} \mathrm{H}-$ NMR $\left(\mathrm{CDCl}_{3}\right) \delta$ ppm: 1.85-2.30 (m, 15H, $\left.5 \mathrm{OAc}\right), 2.35\left(\mathrm{~s}, 3 \mathrm{H}, \mathrm{CH}_{3}\right), 4.00(\mathrm{~m}$, $\left.2 \mathrm{H}, \mathrm{CH}_{2}\right), 4.50-5.50(\mathrm{~m}, 4 \mathrm{H}, 4 \mathrm{CHOAc}), 7.40-7.50(\mathrm{~m}, 5 \mathrm{H}, \mathrm{Ar}-\mathrm{H})$ and 9.30 (br.s, $1 \mathrm{H}, \mathrm{NH}, \mathrm{D}_{2} \mathrm{O}$ exchangeable), ${ }^{13} \mathrm{C}-\mathrm{NMR}$ (DMSO- $\left.d_{6}\right) \delta \mathrm{ppm}: 9.30\left(\mathrm{CH}_{3}\right), 20.5-$ $22.1\left(5 \mathrm{CH}_{3}\right), 64.4\left(\mathrm{CH}_{2}\right), 66.8-72.3(4 \mathrm{CH}), 126.2-133.7$ (Ar-C), 138.5-155.0 (thienopyrimidone carbon atoms and triazole carbon atom) and 169.0-171.1 (6CO); MS (m/z): 642 (68\%). 
3- (1 ,2`3, 4, 5`-0-Pentaacetylmanosyl) -6-methyl-5-oxo-7-phenyl-1,5-dihydrothieno [2,3-d][1,2,4] triazolo[4,3-a]pyrimidine (9b)

A solution of compound $7 \mathrm{~b}(4.34 \mathrm{~g}, 0.01 \mathrm{~mole})$ in a mixture of acetic anhydride-pyridine was stirred to produce $9 \mathrm{~b}$, as deep yellow crystals. IR spectrum $\left(\mathrm{KBr}, \mathrm{cm}^{-1}\right): 3220(\mathrm{NH}), 1745-1720(\mathrm{OAc})$ and $1675\left(\mathrm{CO}\right.$ amide), ${ }^{1} \mathrm{H}-$ NMR $\left(\mathrm{CDCl}_{3}\right) \delta$ ppm: 1.87-2.00 (m, 15H, $\left.5 \mathrm{OAc}\right), 2.45\left(\mathrm{~s}, 3 \mathrm{H}, \mathrm{CH}_{3}\right), 4.00(\mathrm{~m}$, $\left.2 \mathrm{H}, \mathrm{CH}_{2}\right), 5.00-5.55(\mathrm{~m}, 4 \mathrm{H}, 4 \mathrm{CHOAc}), 7.00-7.73(\mathrm{~m}, 5 \mathrm{H}, \mathrm{Ar}-\mathrm{H})$ and $9.20(\mathrm{~s}$, $1 \mathrm{H}, \mathrm{NH}, \mathrm{D}_{2} \mathrm{O}$ exchangeable); MS (m/z): $642(60 \%)$.

3- (1 ,2 ‘3, 4, ‘-0-Tetraacetylarabinosyl) -6-methyl-5-oxo-7-phenyl-1,5-dihydrothieno [2,3-d][1,2,4] triazolo[4,3-a]pyrimidine (9c)

A solution of compound $7 \mathrm{c}(4.04 \mathrm{~g}, 0.01 \mathrm{~mole})$ in a mixture of acetic anhydride-pyridine was stirred to produce 9c, as deep yellow crystals. IR spectrum $\left(\mathrm{KBr}, \mathrm{cm}^{-1}\right): 3215(\mathrm{NH}), 1755-1720(\mathrm{OAc})$ and 1675 (CO amide), ${ }^{1} \mathrm{H}-$ NMR $\left(\mathrm{CDCl}_{3}\right) \delta$ ppm: 1.38-1.59 (m, 12H, $\left.4 \mathrm{OAc}\right), 2.46\left(\mathrm{~s}, 3 \mathrm{H}, \mathrm{CH}_{3}\right), 4.00(\mathrm{~m}$, $\left.2 \mathrm{H}, \mathrm{CH}_{2}\right), 5.00-5.50(\mathrm{~m}, 3 \mathrm{H}, 3 \mathrm{CHOAc}), 7.10-7.46(\mathrm{~m}, 5 \mathrm{H}, \mathrm{Ar}-\mathrm{H})$ and 9.00 (br.s, $1 \mathrm{H}, \mathrm{NH}, \mathrm{D}_{2} \mathrm{O}$ exchangeable), ${ }^{13} \mathrm{C}-\mathrm{NMR}$ (DMSO-d $\left.{ }_{6}\right) \delta \mathrm{ppm}: 9.30\left(\mathrm{CH}_{3}\right), 20.5-$ $21.0\left(4 \mathrm{CH}_{3}\right), 63.5\left(\mathrm{CH}_{2}\right), 67.0-72.3(3 \mathrm{CH}), 126.2-133.7$ (Ar-C), 139.5-155.0 (thienopyrimidone carbon atoms and triazole carbon atom) and 169.8.-170.5 (5CO); MS (m/z): $570(65 \%)$.

Preparation of compounds $10 a-c$

General method

Methanolic sodium methoxide solution (sodium metal $0.23 \mathrm{~g}, 0.01 \mathrm{~mole}$ ) in absolute methanol $(30 \mathrm{ml})$ was added to compound $9 \mathrm{a}-\mathrm{c}(0.01 \mathrm{~mole})$. The reaction mixture was kept under stirring for $6 \mathrm{hr}$, and then neutralized with hydrochloric acid solution and methanol was removed under reduced pressure. The precipitate so-formed was collected by filtration and recrystallized from dioxane to produce $10 \mathrm{a}-\mathrm{c}$.

3-Glucosyl-6-methyl-5-oxo-7-phenyl-1,5-dihydrothieno[2,3-d][1,2,4] triazolo [4,3-a] pyrimidine (10a)

Methanolic sodium methoxide solution was added to compound 9a (6.42 g, 0.01 mole) to produce $10 \mathrm{a}$, as pale yellow crystals. IR spectrum $\left(\mathrm{KBr}, \mathrm{cm}^{-1}\right): 3440,3430$ $(\mathrm{OH}), 3210(\mathrm{NH})$ and 1680(CO amide), ${ }^{1} \mathrm{H}-\mathrm{NMR}$ (DMSO- $\left.d_{6}\right) \delta$ ppm: 2.35 (s, 3H, $\left.\mathrm{CH}_{3}\right), 3.60-3.70\left(\mathrm{~m}, 5 \mathrm{H}, 5 \mathrm{OH}, \mathrm{D}_{2} \mathrm{O}\right.$ exchangeable), $3.95\left(\mathrm{~m}, 2 \mathrm{H}, \mathrm{CH}_{2}\right), 4.00(\mathrm{~m}, 1 \mathrm{H}$ $\left.\mathrm{H}-4^{`}\right), 4.20\left(\mathrm{~m}, 1 \mathrm{H}, \mathrm{H}-2^{`}\right), 4.65$ (d, J=6.45Hz 1H, H-3`), 4.75 (d, J=7.25Hz, $1 \mathrm{H}, \mathrm{H}-$ 1) $)$ 7.40-7.50 (m, 5H, Ar-H) and $11.30\left(\mathrm{~s}, 1 \mathrm{H}, \mathrm{NH}, \mathrm{D}_{2} \mathrm{O}\right.$ exchangeable); ${ }^{13} \mathrm{C}-\mathrm{NMR}$ $\left(\mathrm{DMSO}-d_{6}\right) \delta$ ppm: $9.30\left(\mathrm{CH}_{3}\right), 64.4\left(\mathrm{CH}_{2}\right), 68.2-72.3(4 \mathrm{CH}), 126.4-129.2(\mathrm{Ar}-\mathrm{C})$, 135.5-158.0 (thienopyri-midone carbon atoms triazole carbon atom) and $170.0(\mathrm{CO})$; MS (m/z): $432(51 \%)$.

3-Mannosyl-6-methyl-5-oxo-7-phenyl-1,5-dihydrothieno[2,3-d][1,2,4]triazolo[4,3-a] pyrimidine (10b)

Methanolic sodium methoxide solution was added to compound $9 \mathrm{~b}(6.42 \mathrm{~g}$, 0.01 mole) to produce $10 \mathrm{~b}$, as pale yellow crystals. IR spectrum $\left(\mathrm{KBr}, \mathrm{cm}^{-1}\right)$ : 3430, $3420(\mathrm{OH}), 3115(\mathrm{NH})$ and 1675(CO), ${ }^{1} \mathrm{H}-\mathrm{NMR}\left(\mathrm{DMSO}-d_{6}\right) \delta$ ppm: 2.40 
(s, 3H, $\left.\mathrm{CH}_{3}\right), 3.50-3.58\left(\mathrm{~m}, 5 \mathrm{H}, 5 \mathrm{OH}, \mathrm{D}_{2} \mathrm{O}\right.$-exchangeable), $3.90\left(\mathrm{~m}, 2 \mathrm{H}, \mathrm{CH}_{2}\right)$, 4.35-4.60 (m, 2H, H-4', H-2`), 4.65 (d, J=7.25Hz 1H, H-3`), 5.20 (d, J=8.15Hz, $\left.1 \mathrm{H}, \mathrm{H}-1^{`}\right), 7.30-7.50$ (m, 5H,Ar-H) and 10.35 (br.s, 1H, NH, $\mathrm{D}_{2} \mathrm{O}$ exchangeable); MS (m/z): $420(62 \%)$.

3-Arabinosyl-6-methyl-5-oxo -7-phenyl-1, 5-dihydrothieno [2,3-d] [1,2,4] triazolo [4,3-a] pyrimidine (10c)

Methanolic sodium methoxide solution was added to compound 9c (5.70 g, 0.01 mole) to produce $10 \mathrm{c}$, as pale yellow crystals. IR spectrum $\left(\mathrm{KBr}, \mathrm{cm}^{-1}\right): 3455,3440$ $(\mathrm{OH}), 3210(\mathrm{NH})$ and 1660 (CO amide). ${ }^{1} \mathrm{H}-\mathrm{NMR}$ (DMSO- $\left.d_{6}\right) \delta \mathrm{ppm}: 2.30$ (s, 3H, $\mathrm{CH}_{3}$ ), 3.55-3.70 (m, 4H, $4 \mathrm{OH}, \mathrm{D}_{2} \mathrm{O}$-exchangeable), $3.80\left(\mathrm{~m}, 2 \mathrm{H}, \mathrm{CH}_{2}\right), 4.15-4.25(\mathrm{~m}$, $\left.1 \mathrm{H}, \mathrm{H}-3^{`}\right), 4.30-4.55\left(\mathrm{~m}, 1 \mathrm{H}, \mathrm{H}-2^{\prime}\right), 5.15$ (d, J=7.25Hz, 1H, H-1') 7.35-7.55 (m, $5 \mathrm{H}, \mathrm{Ar}-\mathrm{H})$ and 10.25 (br.s, $1 \mathrm{H}, \mathrm{NH}, \mathrm{D}_{2} \mathrm{O}$ exchangeable); $\mathrm{MS}$ (m/z): 402 (55\%).

One pot preparation of 2-galctosylhydrazino-5-methyl-6-phenylthieno [2,3d]pyrimidine-4(4H)-one (11)

A mixture of compound 1a $(2.72 \mathrm{~g}, 0.01 \mathrm{~mole})$ and D-galctose $(1.80 \mathrm{~g}, 0.01 \mathrm{~mole})$ in boiling dioxane in the presence of catalytic amounts of piperidine was stirred under reflux for $5 \mathrm{hrs}$. The precipitate so-formed was collected by filtration and recrystallized from dioxane to produce 11 as yellow crystals. IR spectrum $\left(\mathrm{KBr}, \mathrm{cm}^{-}\right.$ $\left.{ }^{1}\right): 3450,3440(\mathrm{OH}), 3220(\mathrm{NH})$ and $1675(\mathrm{CO}) .{ }^{1} \mathrm{H}-\mathrm{NMR}\left(\mathrm{DMSO}-d_{6}\right) \delta \mathrm{ppm}: 2.35(\mathrm{~s}$, $\left.3 \mathrm{H}, \mathrm{CH}_{3}\right), 3.58-3.65\left(\mathrm{~m}, 5 \mathrm{H}, 5 \mathrm{OH}, \mathrm{D}_{2} \mathrm{O}\right.$-exchangeable), $3.81\left(\mathrm{~m}, 2 \mathrm{H}, \mathrm{CH}_{2}\right), 4.29(\mathrm{~m}$, $\left.1 \mathrm{H}, \mathrm{H}-4^{\prime}\right), 4.36$ (m, 1H, H-2`), $4.60\left(\mathrm{~d}, J=6.5 \mathrm{~Hz}, 1 \mathrm{H}, \mathrm{H}-3^{\circ}\right), 5.15(\mathrm{~d}, J=4.5 \mathrm{~Hz}, 1 \mathrm{H}, \mathrm{H}-$ 1) ) 7.40-7.50 (m, 5H,Ar-H) and 10.30 (br.s, $1 \mathrm{H}, \mathrm{NH}, \mathrm{D}_{2} \mathrm{O}$-exchangeable); ${ }^{13} \mathrm{C}-\mathrm{NMR}$ $\left(\mathrm{DMSO}-d_{6}\right) \delta$ ppm: $9.28\left(\mathrm{CH}_{3}\right), 64.1\left(\mathrm{CH}_{2}\right), 65.2-70.0(4 \mathrm{CH}), 122.4-126.2(\mathrm{Ar}-\mathrm{C})$, 130.5-158.0 (thienopyrimidone carbon atoms and triazole carbon atom) and 169.0 (CO); MS (m/z): 432 (65\%).

\section{Biological evaluation}

Measurement of the antimicrobial activity using Agar diffusion assay method

The antimicrobial activity was determined by the cup-plate-technique method with some modifications to determine the antimicrobial activity of the tested sample. The used medium is nutrient agar and the tested microorganisms are: Gram +ve bacteria \{Staphylococcus aureus, Bacillus subtilis\}, Gram -ve bacteria $\{$ Escherichia coli $\}$, Yeast (single cell fungi) $\{$ Candida albicans $\}$ and Multi-cellular fungi $\{$ Aspergillus niger $\}$. This data was obtained according to the following procedures: The test had been carried out as follows: $40 \mathrm{ml}$ of the medium (at $55-60^{\circ} \mathrm{C}$ ) was inoculated with $200 \mu \mathrm{l}$ of the prepared test microorganism suspensions and poured in $15 \mathrm{~cm}$ diameter plates and mixed well and allowed to solidify. After solidification, holes $(0.9 \mathrm{~cm}$ diameter $)$ were made in the agar plate by the aid of a sterile cork borer. For each sample, we made duplicate holes. In the previously made holes, $50 \mu 1$ of the dissolved sample was placed using an automatic micropipette. The petri-dishes were left at $5^{\circ} \mathrm{C}$ for 1 hour to allow diffusion of the antibiotic through the agar medium prior to the growth of the test organism then incubated at $30^{\circ} \mathrm{C}$ for $24 \mathrm{hr}$. The antimicrobial data are compiled in Table 1. 


\section{Conclusion}

It was possible through the present study to synthesize new fused pyrazolopyrimidine compounds which showed higher antimicrobial activity compared with the standard. Six of the synthesized compounds were found to be highly active against +ve bacteria (staphylococcus aureus $G^{+}$), Staphylococus aureus $G^{+}$), gram -ve bacteria (Bacills subitils $G^{-}$), fungi (Aspergillus flavus) and yeast (Candida albicans). Also, the reaction of 2-hydrazinothienopyrimidone with some aldo-hexoses and aldo-pentoses produced acyclic C- nucleoside compounds.

Acknowledgements: The authors wish to acknowledge their deep thanks and gratitude to Prof. Dr. Ahamed Saleh Ali El-Said, Professor of Photochemistry, Photochemistry Department, National Research Centre, for his continuous advice. Also, the authors are truly thankfull to Department of Microbiology, National Research Center, Egypt for performing the antibacterial and antifungal testing.

\section{References}

1. Huang, Z. and Shi, X., Synthesis, reaction and tautomerism of ketene N,S-acetals with benzothiazoline ring, Chem. Ber. 123, 541 (1990); Chem. Abstr. 112, 158118k (1990).

2. Henriksen, I., Sodium 2-cyanoethylene-1,1-dithiolate tetrahydrate: a stable salt of cyanodithioacetic acid. A New preparative route to 2-cyanoketene $S, S-, S, N$ - and $N, N$ acetals. Acta Chem. Scand. 50, 432 (1996); Chem. Abstr. 125, 86196w (1996).

3. Elgemeie, G.H., Elghandour, A.H., Elzanate, A.M. and Ahmed, S.A., Synthesis of some noval cyanoKetene $S, S$-acetals and their use in heterocyclic synthesis. J. Chem. Soc. Perkin Trans. I, 3285 (1997); Chem. Abstr. 128, 48171v (1998).

4. Tominaga, Y., Luo, J.K. and Castle, R.N., The reaction of methyl 3-amino-4-cyano5-methylthiothiophene-2-carboxylate with dmad. A new synthesis of polyfunctionalized quinolines. J. Heterocycl. Chem. 31, 771 (1994); Chem. Abstr. 121, 255767q (1994).

5. Mashraqui, S.H. and Hariharasubrahmanian, H., Application of commercially available anhydrous potassium fluoride for a convenient synthesis of ketene dithioacetals. J. Chem. Res. (S), 492 (1999); Chem. Abstr. 131, 271477g (1999).

6. Markwalder, J.A. and Arnone, M.R., Synthesis and biological evaluation of 1-aryl4,5-dihydro-1H-pyrazolo[3,4-d]pyrimidin-4-one inhibitors of cyclin-dependent kinases. J. Med. Chem. 47, 5894-5911 (2004).

7. Giovanni, P., El-Kashef, H., Farghaly, A., Vanelle, P. and Fruttarolo, F., Synthesis of new pyrazolo[4,3-e]-1,2,4-triazolo[1,5-c]pyrimidines and related heterocycles. Tetrahedron, 60, 5093-5104 (2004).

8. Mohamed, M.S., Rashad, A.E., Zaki, M.E.A. and Fatahala, S.S., Synthesis and antimicrobial Screening of some fused heterocyclic pyrroles. Acta Pharm. 55, 237-249 (2005).

9. Rashad, A.S., Shamroukh, A.H., Hegab, M.I. and Awad, H.M., Synthesis of some biologically active pyrazoles and c-nucleosides. Acta Chim. Slov. 52, 429-434 (2005).

Egypt. J. Chem. 53, No. 4 (2010) 
10. Fahmi, A.A., Mekki, S.T., Shawali, A.S., Hassaneen, H.M. and Abdelhamid, H., J. Chem. Research (s), 6-7 (1994).

11. Barret, A. and Bronghton, H.A., Direct conversion of unprotected D-ribose into showdomycin and epishowdomycin. J. Org. Chem. 49(19), 3673-3674 (1984).

12. Aslani, S.G., Buchanan, J.G.J., Edgar, A., Shanks, C. and Williams, G., J. Chem. Soc. Perkin Trans. 1, 2267 (1981).

13. Hamed, A., Abo-Amaym, E.R. and El-Ashry, E.H., Nucleosides and Nucleotides, 17(8), 1385-1407 (1998).

14. Abdel Fattah, A.M., Aly, A.S., Abdel Motti, F., Zaki, M.E.A. and Aly, H.A.H. Novel synthesis of some azolothienopyrimidine and pyrimidothienotriazine derivatives, Egypt J. Chem. 38, 635-644 (1995).

15. Abdel Fattah, A.M., Aly, A.S., Abdel Motti, F., Hassan, N.A. and Aly, H.A.H., Synthesis and reaction of some thienopyrimidine derivatives and some of their photooxidation. Egypt J. Chem. 38, 627-633 (1995).

16. A.S.Aly, Kh. M. Abu-Zied and Alaa El-Din M.Gaafar. Synthesis and reactions of some novel azolothienopyrimidines and thienopyrimido-as-triazenes derivatives. Phosphorusm Sulphur, and Silicon, 182,447-474(2007).

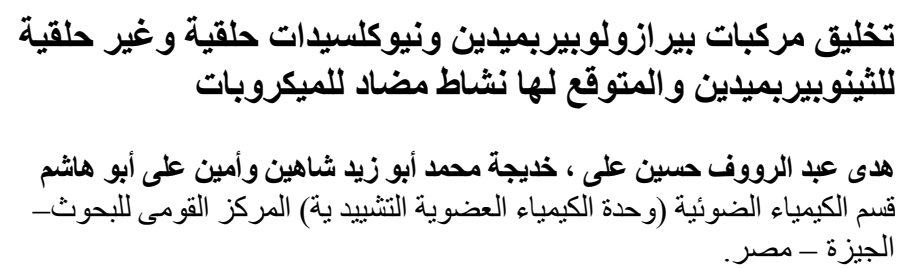

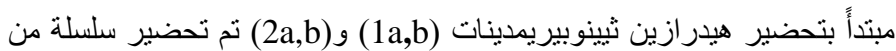

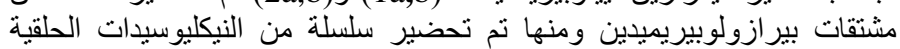

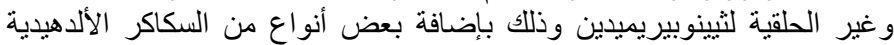

(السداسية و الخماسية) إلى هيدر ازين ثيينو بينيريميدين.

ثم إختبار المواد السابث تحضيرها من ناحية التأثبرات البيولوجية لها على ولى

بعض الفطريات و البكتيريا وقد أعطت نتائج جيدة. 